\title{
Applications of differential subordinations for certain classes of $p$-valent functions associated with generalized Srivastava-Attiya operator
}

\author{
M K Aouf, A O Mostafa, A M Shahin and S M Madian*
}

\section{*Correspondence:}

samar_math@yahoo.com Department of Mathematics,

Faculty of Science, Mansoura University, Mansoura 35516, Egypt

\begin{abstract}
The object of the present paper is to investigate some inclusion relations and other interesting properties for certain classes of $p$-valent functions involving generalized Srivastava-Attiya operator by using the principle of differential subordination.

MSC: $30 C 45$
\end{abstract}

Keywords: differential subordination; integral operator; $p$-valent functions

\section{Introduction}

Let $A(p)$ be the class of functions which are analytic and $p$-valent in the unit disc $U=\{z \in$ $\mathbb{C}:|z|<1\}$ of the form

$$
f(z)=z^{p}+\sum_{k=1}^{\infty} a_{k+p} z^{k+p} \quad(p \in \mathbb{N}=\{1,2, \ldots\}) .
$$

Let also $A(1)=A_{1}$. For $g(z) \in A(p)$, given by $g(z)=z^{p}+\sum_{k=1}^{\infty} b_{k+p} z^{k+p}$, the Hadamard product (or convolution) of $f(z)$ and $g(z)$ is defined by

$$
(f * g)(z)=z^{p}+\sum_{k=1}^{\infty} a_{k+p} b_{k+p} z^{k+p}=(g * f)(z)
$$

Next, in the usual notation, let $\Phi(z, s, a)$ denote the Hurwitz-Lerch Zeta function defined as follows:

$$
\begin{aligned}
& \Phi(z, s, a)=\sum_{k=0}^{\infty} \frac{z^{k}}{(k+a)^{s}} \\
& \quad\left(a \in \mathbb{C} \backslash \mathbb{Z}_{0}^{-}=\{0,-1,-2, \ldots\} ; s \in \mathbb{C} \text { when }|z|<1 ; \operatorname{Re}\{s\}>1 \text { when }|z|=1\right) .
\end{aligned}
$$

For further interesting properties and characteristics of the Hurwitz-Lerch Zeta function $\Phi(z, s, a)$ see $[2,5,8,9,11]$, and [21].

\section{Springer}

๑ 2012 Aouf et al.; licensee Springer. This is an Open Access article distributed under the terms of the Creative Commons Attribution License (http://creativecommons.org/licenses/by/2.0), which permits unrestricted use, distribution, and reproduction in any medium, provided the original work is properly cited. 
Recently, Srivastava and Attiya [20] have introduced the linear operator $L_{s, b}: A_{1} \rightarrow A_{1}$, defined in terms of the Hadamard product by

$$
L_{s, b}(f)(z)=G_{s, b}(z) * f(z) \quad\left(z \in U ; b \in \mathbb{C} \backslash \mathbb{Z}_{0}^{-} ; s \in \mathbb{C}\right),
$$

where

$$
G_{s, b}=(1+b)^{s}\left[\Phi(z, s, b)-b^{-s}\right] \quad(z \in U) .
$$

The Srivastava-Attiya operator $L_{s, b}$ contains, among its special cases, the integral operators introduced and investigated by Alexander [1], Libera [7] and Jung et al. [6].

Analogous to $L_{s, b}$, Liu [10] defined the operator $J_{p, s, b}: A(p) \rightarrow A(p)$ by

$$
J_{p, s, b}(f)(z)=G_{p, s, b}(z) * f(z) \quad\left(z \in U ; b \in \mathbb{C} \backslash \mathbb{Z}_{0}^{-} ; s \in \mathbb{C} ; p \in \mathbb{N}\right),
$$

where

$$
G_{p, s, b}=(1+b)^{s}\left[\Phi_{p}(z, s, b)-b^{-s}\right]
$$

and

$$
\Phi_{p}(z, s, b)=\frac{1}{b^{s}}+\sum_{k=0}^{\infty} \frac{z^{k+p}}{(k+1+b)^{s}} .
$$

It is easy to observe from (1.6) and (1.7) that

$$
J_{p, s, b}(f)(z)=z^{p}+\sum_{k=1}^{\infty}\left(\frac{1+b}{k+1+b}\right)^{s} a_{k+p} z^{k+p} .
$$

We note that

(i) $J_{p, 0, b}(f)(z)=f(z)$;

(ii) $J_{1,1,0}(f)(z)=L f(z)=\int_{0}^{z} \frac{f(t)}{t} d t\left(f \in A_{1}\right)$, where the operator $L$ was introduced by Alexander [1];

(iii) $J_{1, s, b}(f)(z)=L_{s, b} f(z)\left(s \in \mathbb{C}, b \in \mathbb{C} \backslash \mathbb{Z}_{0}^{-}\right)$, where the operator $L_{s, b}$ was introduced by Srivastava-Attiya [20];

(iv) $J_{p, 1, \mu+p-1}(f)(z)=F_{\mu, p}(f)(z)(\mu>-p, p \in \mathbb{N})$, where the operator $F_{\mu, p}$ was introduced by Choi et al. [3];

(v) $J_{p, \alpha, p}(f)(z)=I_{p}^{\alpha} f(z)(\alpha>0, p \in \mathbb{N})$, where the operator $I_{p}^{\alpha}$ was introduced by Shams et al. [18];

(vi) $J_{p, \gamma, p-1}(f)(z)=J_{p}^{\gamma} f(z)\left(\gamma \in \mathbb{N}_{0}=\mathbb{N} \cup\{0\}, p \in \mathbb{N}\right)$, where the operator $J_{p}^{\gamma}$ was introduced by El-Ashwah and Aouf [4];

(vii) $J_{p, \gamma, p+l-1}(f)(z)=J_{p}^{\gamma}(l) f(z)\left(\gamma \in \mathbb{N}_{0}, p \in \mathbb{N}, l \geq 0\right)$, where the operator $J_{p}^{\gamma}(l)$ was introduced by El-Ashwah and Aouf [4].

It follows from (1.8) that

$$
z\left(J_{p, s, b}(f)(z)\right)^{\prime}=(b+1) J_{p, s-1, b}(f)(z)-(b+1-p) J_{p, s, b}(f)(z) .
$$


For two analytic functions $f, g \in A(p)$, we say that $f$ is subordinate to $g$, written $f(z) \prec g(z)$ if there exists a Schwarz function $w(z)$, which (by definition) is analytic in $U$ with $w(0)=0$ and $|w(z)|<1$ for all $z \in U$, such that $f(z)=g(w(z)), z \in U$. Furthermore, if the function $g(z)$ is univalent in $U$, then we have the following equivalence (see [14]):

$$
f(z) \prec g(z) \Leftrightarrow f(0)=g(0) \text { and } f(U) \subset g(U) \text {. }
$$

Definition 1 For fixed parameters $A$ and $B$, with $-1 \leq B<A \leq 1$, we say that $f \in A(p)$ is in the class $S_{p}^{s, b}(A, B)$ if it satisfies the following subordination condition:

$$
\frac{\left(J_{p, s, b}(f)(z)\right)^{\prime}}{p z^{p-1}} \prec \frac{1+A z}{1+B z} \quad(p \in \mathbb{N}) .
$$

In view of the definition of subordination (1.10) is equivalent to the following condition:

$$
\left|\frac{\frac{\left(J_{p, s, b}(f)(z)\right)^{\prime}}{p z^{p-1}}-1}{B \frac{\left(J p_{p, s, b}(f)(z)\right)^{\prime}}{p z^{p-1}}-A}\right|<1 \quad(z \in U) .
$$

For convenience, we write $S_{p}^{s, b}\left(1-\frac{2 \eta}{p},-1\right)=S_{p}^{s, b}(\eta)$, where $S_{p}^{s, b}(\eta)$ denotes the class of functions in $A(p)$ satisfying the inequality

$$
\operatorname{Re}\left(\frac{\left(J_{p, s, b}(f)(z)\right)^{\prime}}{p z^{p-1}}\right)>\eta \quad(0 \leq \eta<1 ; p \in \mathbb{N} ; z \in U) .
$$

In the present paper, we investigate some inclusion relations and other interesting properties for certain classes of $p$-valent functions involving an integral operator.

\section{Preliminaries}

To establish our main results, we need the following lemmas.

Lemma $1([13,14])$ Let $h$ be analytic and convex (univalent) in $U$ with $h(0)=1$. Suppose also that the function $\varphi$ given by

$$
\varphi(z)=1+c_{m} z^{m}+c_{m+1} z^{m+1}+\cdots
$$

is analytic in $U$, where $m$ is a positive integer. If

$$
\varphi(z)+\frac{z \varphi^{\prime}(z)}{\varrho} \prec h(z) \quad(\operatorname{Re}\{\varrho\} \geq 0 ; \varrho \neq 0)
$$

then

$$
\varphi(z) \prec \psi(z)=\frac{\varrho}{m} z^{-\frac{\varrho}{m}} \int_{0}^{z} t^{\frac{\varrho}{m}-1} h(t) d t \prec h(z)
$$

and $\psi(z)$ is the best dominant of (2.2). 
We denote by $H(\varrho)$ the class of functions $\Phi(z)$ given by

$$
\Phi(z)=1+c_{1} z+c_{2} z^{2}+\cdots,
$$

which are analytic in $U$ and satisfy the following inequality:

$$
\operatorname{Re}\{\Phi(z)\}>\varrho \quad(0 \leq \varrho<1 ; z \in U) .
$$

Lemma 2 ([17]) Let the function $\Phi(z) \in H(\varrho)$, where $\Phi(z)$ given by (2.4). Then

$$
\operatorname{Re}\{\Phi(\varrho)\} \geq 2 \varrho-1+\frac{2(1-\varrho)}{1+|z|} \quad(0 \leq \varrho<1 ; z \in U) .
$$

Lemma 3 ([22]) For $0 \leq \varrho_{1}, \varrho_{2}<1$,

$$
H\left(\varrho_{1}\right) * H\left(\varrho_{2}\right) \subset H\left(\varrho_{3}\right), \quad \varrho_{3}=1-2\left(1-\varrho_{1}\right)\left(1-\varrho_{2}\right) .
$$

The result is best possible.

Lemma 4 ([24]) Let $\mu$ be a positive measure on the unit interval [0,1]. Let $g(z, t)$ be a complex valued function defined on $U \times[0,1]$ such that $g(0, t)$ is analytic in $U$ for each $t \in[0,1]$ and such that $g(z, 0)$ is $\mu$ integrable on $[0,1]$ for all $z \in U$. In addition, suppose that $\operatorname{Re}\{g(z, t)\}>0, g(-r, t)$ is real and

$$
\operatorname{Re}\left\{\frac{1}{g(z, t)}\right\} \geq \frac{1}{g(-r, t)} \quad(|z| \leq r<1 ; t \in[0,1]) .
$$

If $G$ is defined by

$$
G(z)=\int_{0}^{1} g(z, t) d \mu(t)
$$

then

$$
\operatorname{Re}\left\{\frac{1}{G(z)}\right\} \geq \frac{1}{G(-r)} \quad(|z| \leq r<1) .
$$

Lemma 5 ([19]) Let the function $g$ be analytic in $U$ with $g(0)=1$ and $\operatorname{Re}\{g(z)\}>\frac{1}{2}(z \in U)$. Then, for any function $F$ analytic in $U,(g * F)(U)$ is contained in the convex hull of $F(U)$.

Lemma 6 ([16]) Let $\varphi$ be analytic in $U$ with $\varphi(0)=1$ and $\varphi(z)=0$ for $0<|z|<1$ and let $A, B \in \mathbb{C}$ with $A \neq B,|B| \leq 1$.

(i) Let $B \neq 0$ and $\gamma \in \mathbb{C}^{*}=\mathbb{C} \backslash\{0\}$ satisfy either $\left|\frac{\gamma(A-B)}{B}-1\right| \leq 1$ or $\left|\frac{\gamma(A-B)}{B}+1\right| \leq 1$. If $\varphi$ satisfies

$$
1+\frac{z \varphi^{\prime}(z)}{\gamma \varphi(z)} \prec \frac{1+A z}{1+B z}
$$

then

$$
\varphi(z) \prec(1+B z)^{\gamma\left(\frac{A-B}{B}\right)},
$$

and this is best dominant. 
(ii) Let $B=0$ and $\gamma \in \mathbb{C}^{*}$ be such that $|\gamma A|<\pi$. If $\varphi$ satisfies (2.5), then

$$
\varphi(z) \prec e^{\gamma A z}
$$

and this is the best dominant.

For real or complex numbers $a, n$ and $c\left(c \notin \mathbb{Z}_{0}^{-}\right)$and $z \in U$, the Gaussian hypergeometric function defined by

$$
\begin{aligned}
{ }_{2} F_{1}(a, n ; c ; z) & =1+\frac{a n}{c} \cdot \frac{z}{1 !}+\frac{a(a+1) n(n+1)}{c(c+1)} \cdot \frac{z^{2}}{2 !}+\cdots \\
& =\sum_{k=0}^{\infty} \frac{(a)_{k}(n)_{k}}{(c)_{k}} \frac{z^{k}}{k !},
\end{aligned}
$$

where $(d)_{k}=d(d+1) \cdots(d+k-1)$ and $(d)_{0}=1$. We note that the series defined by (2.6) converges absolutely for $z \in U$, and hence, ${ }_{2} F_{1}$ represents an analytic function in $U$ (see, for details, [23, Ch.14]).

Lemma 7 ([23]) For real or complex numbers $a, n$ and $c\left(c \notin \mathbb{Z}_{0}^{-}\right)$

$$
\begin{aligned}
& \int_{0}^{1} t^{n-1}(1-t)^{c-n-1}(1-t z)^{-a} d t=\frac{\Gamma(n) \Gamma(c-n)}{\Gamma(c)}{ }_{2} F_{1}(a, n ; c ; z) \quad(\operatorname{Re}\{n\}, \operatorname{Re}\{c\}>0) \\
& { }_{2} F_{1}(a, n ; c ; z)=(1-z)^{-a}{ }_{2} F_{1}\left(a, c-n ; c ; \frac{z}{z-1}\right)
\end{aligned}
$$

and

$$
{ }_{2} F_{1}(a, n ; c ; z)={ }_{2} F_{1}(n, a ; c ; z) .
$$

\section{Main results}

Unless otherwise mentioned, we assume throughout this paper that $-1 \leq B<A \leq 1, s \in \mathbb{C}$, $b \in \mathbb{C} \backslash \mathbb{Z}_{0}^{-}, p \in \mathbb{N} \backslash\{1\}, 0<\alpha \leq 1, m$ is a positive integer and the powers are understood as principle values.

Theorem 1 Let $f$ given by (1.1) satisfy the following subordination condition:

$$
(1-\alpha) \frac{\left(J_{p, s, b}(f)(z)\right)^{\prime}}{p z^{p-1}}+\alpha \frac{\left(J_{p, s, b}(f)(z)\right)^{\prime \prime}}{p(p-1) z^{p-2}} \prec \frac{1+A z}{1+B z}
$$

Then

$$
\frac{\left(J_{p, s, b}(f)(z)\right)^{\prime}}{p z^{p-1}} \prec \Psi(z) \prec \frac{1+A z}{1+B z}
$$

where

$$
\Psi(z)= \begin{cases}\frac{A}{B}+\left(1-\frac{A}{B}\right)(1+B z)^{-1}{ }_{2} F_{1}\left(1,1 ; \frac{p-1}{m \alpha}+1 ; \frac{B z}{1+B z}\right) & \text { for } B \neq 0 \\ 1+\frac{p-1}{m \alpha+p-1} A z & \text { for } B=0\end{cases}
$$


is the best dominant of (3.2). Furthermore,

$$
f \in S_{p}^{s, b}(\beta)
$$

where

$$
\beta= \begin{cases}\frac{A}{B}+\left(1-\frac{A}{B}\right)(1-B)^{-1}{ }_{2} F_{1}\left(1,1 ; \frac{p-1}{m \alpha}+1 ; \frac{B}{B-1}\right) & \text { for } B \neq 0 \\ 1-\frac{p-1}{m \alpha+p-1} A & \text { for } B=0 .\end{cases}
$$

The estimate (3.4) is best possible.

Proof Let

$$
\theta(z)=\frac{\left(J_{p, s, b}(f)(z)\right)^{\prime}}{p z^{p-1}} \quad(z \in U)
$$

where $\theta$ is of the form (2.1) and is analytic in $U$. Differentiating (3.6) with respect to $z$, we get

$$
(1-\alpha) \frac{\left(J_{p, s, b}(f)(z)\right)^{\prime}}{p z^{p-1}}+\alpha \frac{\left(J_{p, s, b}(f)(z)\right)^{\prime \prime}}{p(p-1) z^{p-2}}=\theta(z)+\frac{\alpha}{p-1} z \theta^{\prime}(z) \prec \frac{1+A z}{1+B z}
$$

Applying Lemma 1 for $\varrho=\frac{p-1}{\alpha}$ and Lemma 7, we have

$$
\begin{aligned}
\frac{\left(J_{p, s, b}(f)(z)\right)^{\prime}}{p z^{p-1}} & \prec \Psi(z)=\frac{p-1}{m \alpha} z^{-\frac{p-1}{m \alpha}} \int_{0}^{z} t^{\frac{p-1}{m \alpha}-1}\left(\frac{1+A t}{1+B t}\right) d t \\
& = \begin{cases}\frac{A}{B}+\left(1-\frac{A}{B}\right)(1+B z)^{-1}{ }_{2} F_{1}\left(1,1 ; \frac{p-1}{m \alpha}+1 ; \frac{B z}{1+B z}\right) & \text { for } B \neq 0, \\
1+\frac{p-1}{m \alpha+p-1} A z & \text { for } B=0 .\end{cases}
\end{aligned}
$$

This proves the assertion (3.2) of Theorem 1. Next, in order to prove the assertion (3.4) of Theorem 1, it suffices to show that

$$
\inf _{|z|<1}\{\operatorname{Re}(\Psi(z))\}=\Psi(-1) .
$$

Indeed, we have

$$
\operatorname{Re}\left\{\frac{1+A z}{1+B z}\right\} \geq \frac{1-A r}{1-B r} \quad(|z| \leq r<1) .
$$

Setting

$$
G(z, \zeta)=\frac{1+A \zeta z}{1+B \zeta z} \quad \text { and } \quad d \nu(\zeta)=\frac{p-1}{m \alpha} \zeta^{\frac{p-1}{m \alpha}-1} d \zeta \quad(0 \leq \zeta \leq 1)
$$

which is a positive measure on the closed interval $[0,1]$, we get

$$
\Psi(z)=\int_{0}^{1} G(z, \zeta) d v(\zeta)
$$


Then

$$
\operatorname{Re}\{\Psi(z)\} \geq \int_{0}^{1} \frac{1-A \zeta r}{1-B \zeta r} d v(\zeta)=\Psi(-r) \quad(|z| \leq r<1) .
$$

Letting $r \rightarrow 1^{-}$in the above inequality, we obtain the assertion (3.4). Finally, the estimate (3.4) is best possible as $\Psi$ is the best dominant of (3.2). This completes the proof of Theorem 1.

Theorem 2 Iff $\in S_{p}^{s, b}(\eta)(0 \leq \eta<1)$, then

$$
\operatorname{Re}\left\{(1-\alpha) \frac{\left(J_{p, s, b}(f)(z)\right)^{\prime}}{p z^{p-1}}+\alpha \frac{\left(J_{p, s, b}(f)(z)\right)^{\prime \prime}}{p(p-1) z^{p-2}}\right\}>\eta \quad(|z|<R),
$$

where

$$
R=\left\{\frac{\sqrt{(p-1)^{2}+(m \alpha)^{2}}-m \alpha}{p-1}\right\}^{\frac{1}{m}} .
$$

The result is best possible.

Proof Let $f \in S_{p}^{s, b}(\eta)$, then we write

$$
\frac{\left(J_{p, s, b}(f)(z)\right)^{\prime}}{p z^{p-1}}=\eta+(1-\eta) u(z) \quad(z \in U),
$$

where $u$ is of the form (2.1), is analytic in $U$ and has a positive real part in $U$. Differentiating (3.8) with respect to $z$, we have

$$
\frac{1}{1-\eta}\left\{(1-\alpha) \frac{\left(J_{p, s, b}(f)(z)\right)^{\prime}}{p z^{p-1}}+\alpha \frac{\left(J_{p, s, b}(f)(z)\right)^{\prime \prime}}{p(p-1) z^{p-2}}-\eta\right\}=u(z)+\frac{\alpha}{p-1} z u^{\prime}(z) .
$$

Applying the following well-known estimate [12]:

$$
\frac{\left|z u^{\prime}(z)\right|}{\operatorname{Re}\{u(z)\}} \leq \frac{2 m r^{m}}{1-r^{2 m}} \quad(|z|=r<1)
$$

in (3.9), we have

$$
\begin{aligned}
& \frac{1}{1-\eta} \operatorname{Re}\left\{(1-\alpha) \frac{\left(J_{p, s, b}(f)(z)\right)^{\prime}}{p z^{p-1}}+\alpha \frac{\left(J_{p, s, b}(f)(z)\right)^{\prime \prime}}{p(p-1) z^{p-2}}-\eta\right\} \\
& \geq \operatorname{Re}\{u(z)\}\left(1-\frac{2 \alpha m r^{m}}{(p-1)\left[1-r^{2 m}\right]}\right),
\end{aligned}
$$

such that the right-hand side of (3.10) is positive, if $r<R$, where $R$ is given by (3.7).

In order to show that the bound $R$ is best possible, we consider the function $f \in A(p)$ defined by

$$
\frac{\left(J_{p, s, b}(f)(z)\right)^{\prime}}{p z^{p-1}}=\eta+(1-\eta) \frac{1+z^{m}}{1-z^{m}} \quad(0 \leq \eta<1 ; z \in U) .
$$


Note that

$$
\frac{1}{1-\eta}\left\{(1-\alpha) \frac{\left(J_{p, s, b}(f)(z)\right)^{\prime}}{p z^{p-1}}+\alpha \frac{\left(J_{p, s, b}(f)(z)\right)^{\prime \prime}}{p(p-1) z^{p-2}}-\eta\right\}=\frac{(p-1)\left(1-z^{2 m}\right)-2 \alpha m z^{m}}{(p-1)\left(1-z^{m}\right)^{2}}=0,
$$

for $z=R \cdot \exp \left\{\frac{\mathrm{i} \pi}{\mathbf{m}}\right\}$. This completes the proof of Theorem 2 .

For a function $f \in A(p)$, the generalized Bernardi-Libera-Livingston integral operator $F_{\mu, p}$ is defined by

$$
\begin{aligned}
F_{\mu, p}(f)(z) & =\frac{\mu+p}{z^{\mu}} \int_{0}^{z} t^{\mu-1} f(t) d t=\left(z^{p}+\sum_{k=1}^{\infty} \frac{\mu+p}{\mu+p+k} z^{k+p}\right) * f(z) \\
& =z^{p}{ }_{2} F_{1}(1, \mu+p ; \mu+p+1 ; z) * f(z) \quad(\mu>-p ; z \in U) .
\end{aligned}
$$

From (1.8) and (3.11), we have

$$
z\left(J_{p, s, b} \mathrm{~F}_{\mu, p}(f(z))\right)^{\prime}=(\mu+p) J_{p, s, b}(f)(z)-\mu J_{p, s, b} \mathrm{~F}_{\mu, p}(f(z)) \quad(\mu>-p ; z \in U)
$$

and

$$
J_{p, s, b} \mathrm{~F}_{\mu, p}(f(z))=\mathrm{F}_{\mu, p}\left(J_{p, s, b}(f)(z)\right) .
$$

Theorem 3 Let $f \in S_{p}^{s, b}(A, B)$ and $F_{\mu, p}$ be defined by (3.11). Then

$$
\frac{\left(J_{p, s, b} \mathrm{~F}_{\mu, p}(f(z))\right)^{\prime}}{p z^{p-1}} \prec \Phi(z) \prec \frac{1+A z}{1+B z}
$$

where

$$
\Phi(z)= \begin{cases}\frac{A}{B}+\left(1-\frac{A}{B}\right)(1+B z)^{-1} F_{1}\left(1,1 ; \frac{\mu+p}{m}+1 ; \frac{B z}{1+B z}\right) & \text { for } B \neq 0, \\ 1+\frac{\mu+p}{\mu+p+m} A z & \text { for } B=0\end{cases}
$$

is the best dominant of (3.13). Furthermore,

$$
\operatorname{Re}\left\{\frac{\left(J_{p, s, b} \mathrm{~F}_{\mu, p}(f(z))\right)^{\prime}}{p z^{p-1}}\right\}>\psi \quad(z \in U)
$$

where

$$
\psi= \begin{cases}\frac{A}{B}+\left(1-\frac{A}{B}\right)(1-B)_{2}^{-1} F_{1}\left(1,1 ; \frac{\mu+p}{m}+1 ; \frac{B}{B-1}\right) & \text { for } B \neq 0, \\ 1-\frac{\mu+p}{\mu+p+m} A & \text { for } B=0 .\end{cases}
$$

The result is best possible.

Proof Let

$$
K(z)=\frac{\left(J_{p, s, b} \mathrm{~F}_{\mu, p}(f(z))\right)^{\prime}}{p z^{p-1}} \quad(z \in U)
$$


where $K$ is of the form (2.1) and is analytic in $U$. Using (3.12) in (3.15) and differentiating the resulting equation with respect to $z$, we have

$$
\frac{\left(J_{p, s, b}(f)(z)\right)^{\prime}}{p z^{p-1}}=K(z)+\frac{z K^{\prime}(z)}{p+\mu} \prec \frac{1+A z}{1+B z} .
$$

The remaining part of the proof is similar to that of Theorem 1, and so we omit it.

We note that

$$
\frac{\left(J_{p, s, b} \mathrm{~F}_{\mu, p}(f(z))\right)^{\prime}}{p z^{p-1}}=\frac{p+\mu}{p z^{p+\mu}} \int_{0}^{z} t^{\mu}\left(J_{p, s, b}(f)(t)\right)^{\prime} d t \quad(f \in A(p) ; z \in U) .
$$

Putting $A=1-\frac{2 \delta}{p}(0 \leq \delta<1)$ and $B=-1$ in Theorem 3 and using (3.16), we obtain the following corollary.

Corollary 1 If $\in A(p)$ satisfies the following inequality:

$$
\operatorname{Re}\left\{\frac{\left(J_{p, s, b} f(z)\right)^{\prime}}{p z^{p-1}}\right\}>\delta \quad(0 \leq \delta<1 ; z \in U)
$$

then

$$
\operatorname{Re}\left\{\frac{p+\mu}{p z^{p+\mu}} \int_{0}^{z} t^{\mu}\left(J_{p, s, b}(f)(t)\right)^{\prime} d t\right\}>\frac{\delta}{p}+\left(1-\frac{\delta}{p}\right)\left[{ }_{2} F_{1}\left(1,1 ; \frac{\mu+p}{m}+1 ; \frac{1}{2}\right)-1\right] \quad(z \in U) .
$$

The result is best possible.

Theorem 4 Let $f, g \in A(p)$ satisfy the following inequality:

$$
\operatorname{Re}\left\{\frac{J_{p, s, b}(g)(z)}{z^{p}}\right\}>0 \quad(z \in U)
$$

If

$$
\left|\frac{J_{p, s, b}(f)(z)}{J_{p, s, b}(g)(z)}-1\right|<1 \quad(z \in U)
$$

then

$$
\operatorname{Re}\left\{\frac{z\left(J_{p, s, b}(f)(z)\right)^{\prime}}{J_{p, s, b}(f)(z)}\right\}>0 \quad(|z|<\stackrel{\circ}{R})
$$

where

$$
\stackrel{R}{R}=\left(\frac{-3 m+\sqrt{9 m^{2}+4 p(p+m)}}{2(p+m)}\right)^{\frac{1}{m}} .
$$

Proof Let

$$
q(z)=\frac{J_{p, s, b}(f)(z)}{J_{p, s, b}(g)(z)}-1=c_{m} z^{m}+c_{m+1} z^{m+1}+\cdots,
$$


where $q(z)$ is analytic in $U$ with $q(0)=0$ and $|q(z)| \leq|z|^{m}$. Then, by applying the familiar Schwartz Lemma [15], we have $q(z)=z^{m} \mathcal{X}(z)$, where $\mathcal{X}$ is analytic in $U$ and $|\mathcal{X}(z)| \leq 1$. Therefore (3.18) leads to

$$
J_{p, s, b}(f)(z)=J_{p, s, b}(g)(z)\left(1+z^{m} \mathcal{X}(z)\right) \quad(z \in U)
$$

Differentiating (3.19) logarithmically with respect to $z$, we have

$$
\frac{z\left(J_{p, s, b}(f)(z)\right)^{\prime}}{J_{p, s, b}(f)(z)}=\frac{z\left(J_{p, s, b}(g)(z)\right)^{\prime}}{J_{p, s, b}(g)(z)}+\frac{z^{m}\left\{m \mathcal{X}(z)+z \mathcal{X}^{\prime}(z)\right\}}{1+z^{m} \mathcal{X}(z)} .
$$

Letting

$$
\omega(z)=\frac{J_{p, s, b}(g)(z)}{z^{p}} \quad(z \in U)
$$

where $\omega$ is in the form (2.1), is analytic in $U, \operatorname{Re}\{\omega(z)\}>0$ and

$$
\frac{z\left(J_{p, s, b}(g)(z)\right)^{\prime}}{J_{p, s, b}(g)(z)}=\frac{z \omega^{\prime}(z)}{\omega(z)}+p
$$

then we have

$$
\operatorname{Re}\left\{\frac{z\left(J_{p, s, b}(f)(z)\right)^{\prime}}{J_{p, s, b}(f)(z)}\right\} \geq p-\left|\frac{z \omega^{\prime}(z)}{\omega(z)}\right|-\left|\frac{z^{m}\left\{m \mathcal{X}(z)+z \mathcal{X}^{\prime}(z)\right\}}{1+z^{m} \mathcal{X}(z)}\right|
$$

Using the following known estimates [12] (see also [15]):

$$
\left|\frac{\omega^{\prime}(z)}{\omega(z)}\right| \leq \frac{2 m r^{m-1}}{1-r^{2 m}} \quad \text { and } \quad\left|\frac{m \mathcal{X}(z)+z \mathcal{X}^{\prime}(z)}{1+z^{m} \mathcal{X}(z)}\right| \leq \frac{m}{1-r^{m}} \quad(|z|=r<1)
$$

in (3.21), we have

$$
\operatorname{Re}\left\{\frac{z\left(J_{p, s, b}(f)(z)\right)^{\prime}}{J_{p, s, b}(f)(z)}\right\} \geq \frac{p-3 m r^{m}-(p+m) r^{2 m}}{1-r^{2 m}} \quad(|z|=r<1)
$$

which is certainly positive, provided that $r<\stackrel{\circ}{R}$, where $\stackrel{\circ}{R}$ is given by (3.17). This completes the proof of Theorem 4 .

Theorem 5 Let $-1 \leq B_{i}<A_{i} \leq 1(i=1,2)$ and $\tau<p$. If each of the functions $f_{i} \in A(p)$ satisfies the following subordination condition:

$$
(1-\alpha) \frac{\left(J_{p, s, b}\left(f_{i}\right)(z)\right)^{\prime}}{p z^{p-1}}+\alpha \frac{\left(J_{p, s, b}\left(f_{i}\right)(z)\right)^{\prime \prime}}{p(p-1) z^{p-2}} \prec \frac{1+A_{i} z}{1+B_{i} z} \quad(i=1,2),
$$

then

$$
(1-\alpha) \frac{\left(J_{p, s, b}(F)(z)\right)^{\prime}}{p z^{p-1}}+\alpha \frac{\left(J_{p, s, b}(F)(z)\right)^{\prime \prime}}{p(p-1) z^{p-2}} \prec \frac{1+\left(1-\frac{2 \tau}{p}\right) z}{1-z}
$$


where

$$
F(z)=J_{p, s, b}\left(f_{1} * f_{2}\right)(z)
$$

and

$$
\tau=p-4 p \frac{\left(A_{1}-B_{1}\right)\left(A_{2}-B_{2}\right)}{\left(1-B_{1}\right)\left(1-B_{2}\right)}\left[1-\frac{1}{2}{ }_{2} F_{1}\left(1,1 ; \frac{p-1}{\alpha}+1 ; \frac{1}{2}\right)\right] .
$$

The result is best possible when $B_{1}=B_{2}=-1$.

Proof Suppose that the functions $f_{i} \in A(p)(i=1,2)$ satisfy the condition (3.22). Then by setting

$$
h_{i}(z)=(1-\alpha) \frac{\left(J_{p, s, b}\left(f_{i}\right)(z)\right)^{\prime}}{p z^{p-1}}+\alpha \frac{\left(J_{p, s, b}\left(f_{i}\right)(z)\right)^{\prime \prime}}{p(p-1) z^{p-2}} \quad(i=1,2),
$$

we have

$$
h_{i} \in H\left(\varrho_{i}\right), \quad \varrho_{i}=\frac{1-A_{\mathbf{i}}}{1-B_{\mathbf{i}}}(i=1,2) .
$$

And

$$
\left(J_{p, s, b}\left(f_{\mathbf{i}}\right)(z)\right)^{\prime}=\frac{p(p-1)}{\alpha} z^{\frac{(1-p)(1-\alpha)}{\alpha}} \int_{0}^{z} t^{\frac{p-1}{\alpha}-1} h_{i}(t) d t \quad(i=1,2),
$$

from (3.24), (3.26) and (3.27), we have

$$
\left(J_{p, s, b}(F)(z)\right)^{\prime}=\frac{p(p-1)}{\alpha} \mathbf{z}^{\frac{(1-\mathbf{p})(1-\alpha)}{\alpha}} \int_{0}^{z} t^{\frac{p-1}{\alpha}-1} H(t) d t \quad(i=1,2) .
$$

For convenience,

$$
\begin{aligned}
H(z) & =(1-\alpha) \frac{\left(J_{p, s, b}(F)(z)\right)^{\prime}}{p z^{p-1}}+\alpha \frac{\left(J_{p, s, b}(F)(z)\right)^{\prime \prime}}{p(p-1) z^{p-2}} \\
& =\frac{p(p-1)}{\alpha} z^{\frac{(1-p)}{\alpha}} \int_{0}^{z} t^{\frac{p-1}{\alpha}-1}\left(h_{1} * h_{2}\right)(t) d t .
\end{aligned}
$$

Since $h_{i} \in H\left(\varrho_{i}\right)(i=1,2)$, it follows from Lemma 3 that

$$
\left(h_{1} * h_{2}\right)(z) \in H\left(\varrho_{3}\right), \quad \varrho_{3}=1-2\left(1-\varrho_{1}\right)\left(1-\varrho_{2}\right) .
$$

By using (3.30) in (3.29) and applying Lemmas 2 and 3, we have

$$
\begin{aligned}
\operatorname{Re}\{H(z)\} & =\frac{p(p-1)}{\alpha} \int_{0}^{1} s^{\frac{p-1}{\alpha}-1} \operatorname{Re}\left\{\left(h_{1} * h_{2}\right)(s z)\right\} d s \\
& \geq \frac{p(p-1)}{\alpha} \int_{0}^{1} s^{\frac{p-1}{\alpha}-1}\left(2 \varrho_{3}-1+\frac{2\left(1-\varrho_{3}\right)}{1+s|z|}\right) d s \\
& >\frac{p(p-1)}{\alpha} \int_{0}^{1} s^{\frac{p-1}{\alpha}-1}\left(2 \varrho_{3}-1+\frac{2\left(1-\varrho_{3}\right)}{1+s}\right) d s
\end{aligned}
$$




$$
\begin{aligned}
& =p-4 p \frac{\left(A_{1}-B_{1}\right)\left(A_{2}-B_{2}\right)}{\left(1-B_{1}\right)\left(1-B_{2}\right)}\left[1-\frac{p-1}{\alpha} \int_{0}^{1} s^{\frac{p-1}{\alpha}-1}(1+s)^{-1} d s\right] \\
& =p-4 p \frac{\left(A_{1}-B_{1}\right)\left(A_{2}-B_{2}\right)}{\left(1-B_{1}\right)\left(1-B_{2}\right)}\left[1-\frac{1}{2}{ }_{2} F_{1}\left(1,1 ; \frac{p-1}{\alpha}+1 ; \frac{1}{2}\right)\right] \quad(\mathbf{z} \rightarrow-\mathbf{1}) \\
& =\tau \quad(z \in U) .
\end{aligned}
$$

When $B_{1}=B_{2}=-1$, we consider $f_{i} \in A(p)(i=1,2)$ satisfy the condition (3.22) and are defined by

$$
\left(J_{p, s, b}\left(f_{i}\right)(z)\right)^{\prime}=\frac{p(p-1)}{\alpha} z^{\frac{(1-p)(1-\alpha)}{\alpha}} \int_{0}^{z} t^{\frac{p-1}{\alpha}-1}\left(\frac{1+A_{i} t}{1-t}\right) d t \quad(i=1,2) .
$$

By using (3.29) and applying Lemma 3, we have

$$
\begin{aligned}
H(z) & =\frac{p(p-1)}{\alpha} \int_{0}^{1} s^{\frac{p-1}{\alpha}-1}\left[1-\left(1+A_{1}\right)\left(1+A_{2}\right)+\frac{\left(1+A_{1}\right)\left(1+A_{2}\right)}{1-s z}\right] d s \\
& =p-p\left(1+A_{1}\right)\left(1+A_{2}\right)+p\left(1+A_{1}\right)\left(1+A_{2}\right)(1-z)^{-1}{ }_{2} F_{1}\left(1,1 ; \frac{p-1}{\alpha}+1 ; \frac{z}{z-1}\right) \\
& \rightarrow p-p\left(1+A_{1}\right)\left(1+A_{2}\right)+\frac{p}{2}\left(1+A_{1}\right)\left(1+A_{2}\right)_{2} F_{1}\left(1,1 ; \frac{p-1}{\alpha}+1 ; \frac{1}{2}\right) \quad(z \rightarrow-1) .
\end{aligned}
$$

This completes the proof of Theorem 5.

Remark 1 Putting $A_{i}=1-2 \theta_{i}\left(0 \leq \theta_{i}<1\right)$ and $B_{i}=-1(i=1,2)$ in Theorem 5 , we obtain the result obtained by Liu [10, Theorem 5$]$.

Putting $A_{i}=1-2 \theta_{i}\left(0 \leq \theta_{i}<1\right), B_{i}=-1(i=1,2)$ and $s=0$ in Theorem 5 , we obtain the following corollary.

Corollary 2 Let $\chi<p$ and $f_{i} \in A(p)$ satisfy the following inequality:

$$
\operatorname{Re}\left\{(1-\alpha) \frac{f_{i}^{\prime}(z)}{p z^{p-1}}+\alpha \frac{f_{i}^{\prime \prime}(z)}{p(p-1) z^{p-2}}\right\}>\theta_{i} \quad\left(0 \leq \theta_{i}<1 ; i=1,2\right)
$$

then

$$
\operatorname{Re}\left\{(1-\alpha) \frac{\left(f_{1} * f_{2}\right)^{\prime}(z)}{p z^{p-1}}+\alpha \frac{\left(f_{1} * f_{2}\right)^{\prime \prime}(z)}{p(p-1) z^{p-2}}\right\}>\frac{\chi}{p}
$$

where

$$
\chi=p-4 p\left(1-\theta_{1}\right)\left(1-\theta_{2}\right)\left[1-\frac{1}{2}{ }_{2} F_{1}\left(1,1 ; \frac{p-1}{\alpha}+1 ; \frac{1}{2}\right)\right] .
$$

The result is best possible.

Theorem 6 Let $f \in S_{p}^{s, b}(A, B)$ and $g \in A(p)$ satisfy the following inequality:

$$
\operatorname{Re}\left\{\frac{g(z)}{z^{p}}\right\}>\frac{1}{2} \quad(z \in U)
$$


then

$$
(f * g)(z) \in S_{p}^{s, b}(A, B)
$$

Proof We have

$$
\frac{\left(J_{p, s, b}(f * g)(z)\right)^{\prime}}{p z^{p-1}}=\frac{\left(J_{p, s, b}(f)(z)\right)^{\prime}}{p z^{p-1}} * \frac{g(z)}{z^{p}} \quad(z \in U)
$$

where $g(z)$ satisfies (3.31) and $\frac{1+A z}{1+B z}$ is convex (univalent) in $U$. By using (1.10) and applying Lemma 5, we complete the proof of Theorem 6.

Theorem 7 Let $\sigma>0$ and $f \in A(p)$ satisfy the following subordination condition:

$$
(1-\alpha) \frac{J_{p, s, b}(f)(z)}{z^{p}}+\alpha \frac{\left(J_{p, s, b}(f)(z)\right)^{\prime}}{p z^{p-1}} \prec \frac{1+A z}{1+B z} .
$$

Then

$$
\operatorname{Re}\left\{\frac{J_{p, s, b}(f)(z)}{z^{p}}\right\}^{\frac{1}{\sigma}}>\gamma^{\frac{1}{\sigma}}
$$

where

$$
\gamma= \begin{cases}\frac{A}{B}+\left(1-\frac{A}{B}\right)(1-B)_{2}^{-1} F_{1}\left(1,1 ; \frac{p}{m \alpha}+1 ; \frac{B}{B-1}\right) & \text { for } B \neq 0, \\ 1-\frac{p}{m \alpha+p} A & \text { for } B=0 .\end{cases}
$$

The result is best possible.

Proof Let

$$
M(z)=\frac{J_{p, s, b}(f)(z)}{z^{p}} \quad(z \in U)
$$

where $M$ is of the form (2.1) and is analytic in $U$. Differentiating (3.33) with respect to $z$, we have

$$
(1-\alpha) \frac{J_{p, s, b}(f)(z)}{z^{p}}+\alpha \frac{\left(J_{p, s, b}(f)(z)\right)^{\prime}}{p z^{p-1}}=M(z)+\frac{\alpha}{p} z M^{\prime}(z) \prec \frac{1+A z}{1+B z} .
$$

Now, by following steps similar to the proof of Theorem 1 and using the elementary inequality

$$
\operatorname{Re}\left\{\Upsilon^{1 / \varkappa}\right\} \geq\{\operatorname{Re} \Upsilon\}^{1 / \varkappa} \quad(\operatorname{Re}\{\Upsilon\}>0 ; \varkappa \in \mathbb{N})
$$

we obtain the result asserted by Theorem 7 . 
Theorem 8 Let $v \in \mathbb{C}^{*}$ and $A, B \in \mathbb{C}$ with $A \neq B$ and $|B| \leq 1$. Suppose that

$$
\begin{aligned}
& \left|\frac{v(b+1)(A-B)}{B}-1\right| \leq 1 \quad \text { or } \quad\left|\frac{v(b+1)(A-B)}{B}+1\right| \leq 1, \quad \text { if } B \neq 0, \\
& |(b+1) \nu A| \leq \pi, \quad \text { if } B=0 .
\end{aligned}
$$

If $\in A(p)$ with $J_{p, s, b}(f)(z) \neq 0$ for all $z \in U^{*}=U \backslash\{0\}$, then

$$
\frac{J_{p, s-1, b}(f)(z)}{J_{p, s, b}(f)(z)} \prec \frac{1+A z}{1+B z},
$$

implies

$$
\left(\frac{J_{p, s, b}(f)(z)}{z^{p}}\right)^{v} \prec q_{1}(z)
$$

where

$$
q_{1}(z)= \begin{cases}(1+B z)^{\nu(b+1)(A-B) / B}, & \text { if } B \neq 0, \\ e^{\nu(b+1) A z}, & \text { if } B=0,\end{cases}
$$

is the best dominant.

Proof Let us put

$$
\varphi(z)=\left(\frac{J_{p, s, b}(f)(z)}{z^{p}}\right)^{v} \quad(z \in U) .
$$

Then $\varphi$ is analytic in $U, \varphi(0)=1$ and $\varphi(z) \neq 0$ for all $z \in U$. Taking the logarithmic derivatives in both sides of (3.34) and using the identity (1.9), we have

$$
1+\frac{z \varphi^{\prime}(z)}{v(b+1) \varphi(z)}=\frac{J_{p, s-1, b}(f)(z)}{J_{p, s, b}(f)(z)} \prec \frac{1+A z}{1+B z} .
$$

Now the assertions of Theorem 8 follow by using Lemma 6 for $\gamma=v(b+1)$.

Putting $B=-1$ and $A=1-2 \sigma, 0 \leq \sigma<1$, in Theorem 8, we obtain the following corollary.

Corollary 3 Assume that $v \in \mathbb{C}^{*}$ satisfies either $|2 v(b+1)(1-\sigma)-1| \leq 1$ or $\mid 2 v(b+1)(1-$ $\sigma)+1 \mid \leq 1$. Iff $\in A(p)$ with $J_{p, s, b}(f)(z) \neq 0$ for $z \in U^{*}$, then

$$
\operatorname{Re}\left\{\frac{J_{p, s-1, b}(f)(z)}{J_{p, s, b}(f)(z)}\right\}>\sigma \quad(z \in U)
$$

implies

$$
\left(\frac{J_{p, s, b}(f)(z)}{z^{p}}\right)^{v} \prec q_{2}(z)=(1-z)^{-2 v(b+1)(1-\sigma)},
$$

and $q_{2}$ is the best dominant. 
Remark 2 Specializing the parameters $s$ and $b$ in the above results of this paper, we obtain the results for the corresponding operators $F_{\mu, p}, I_{p}^{\alpha}, J_{p}^{\gamma}$ and $J_{p}^{\gamma}(l)$ which are defined in the introduction.

\section{Competing interests}

The authors declare that they have no competing interests.

Authors' contributions

All authors read and approved the final manuscript.

\section{Acknowledgements}

The authors would like to thank the referees of the paper for their helpful suggestions.

Received: 19 December 2011 Accepted: 21 June 2012 Published: 5 July 2012

\section{References}

1. Alexander, JW: Functions which map the interior of the unit circle upon simple regions. Ann. Math., Ser. 2 17, 12-22 (1915)

2. Choi, J, Srivastava, HM: Certain families of series associated with the Hurwitz-Lerch Zeta function. Appl. Math. Comput. 170, 399-409 (2005)

3. Choi, JH, Saigo, M, Srivastava, HM: Some inclusion properties of a certain family of integral operators. J. Math. Anal. Appl. 276, 432-445 (2002)

4. El-Ashwah, RM, Aouf, MK: Some properties of new integral operator. Acta Univ. Apulensis, Mat.-Inform. 24, 51-61 (2010)

5. Ferreira, C, Lopez, JL: Asymptotic expansions of the Hurwitz-Lerch Zeta function. J. Math. Anal. Appl. 298, 210-224 (2004)

6. Jung, IB, Kim, YC, Srivastava, HM: The Hardy space of analytic functions associated with certain one parameter families of integral operators. J. Math. Anal. Appl. 176, 138-147 (1993)

7. Libera, RJ: Some classes of regular univalent functions. Proc. Am. Math. Soc. 16, 755-758 (1969)

8. Lin, S-D, Srivastava, HM: Some families of the Hurwitz-Lerch Zeta functions and associated fractional derivative and other integral representations. Appl. Math. Comput. 154, 725-733 (2004)

9. Lin, S-D, Srivastava, HM, Wang, P-Y: Some expansion formulas for a class of generalized Hurwitz-Lerch Zeta functions. Integral Transforms Spec. Funct. 17, 817-827 (2006)

10. Liu, J-L: Subordinations for certain multivalent analytic functions associated with the generalized Srivastava-Attiya operator. Integral Transforms Spec. Funct. 18, 207-216 (2007)

11. Luo, Q-M, Srivastava, HM: Some generalizations of the Apostol-Bernoulli and Apostol-Euler polynomials. J. Math. Anal. Appl. 308, 290-302 (2005)

12. MacGregor, TH: Radius of univalence of certain analytic functions. Proc. Am. Math. Soc. 14, 514-520 (1963)

13. Miller, SS, Mocanu, PT: Differential subordinations and univalent functions. Mich. Math. J. 28, 157-171 (1981)

14. Miller, SS, Mocanu, PT: Differential Subordination. Theory and Applications. Monographs and Textbooks in Pure and Applied Mathematics, vol. 225. Marcel Dekker, New York (2000)

15. Nehari, PT: Conformal Mapping. McGraw-Hill, New York (1952)

16. Obradovic, M, Owa, S: On certain properties for some classes of starlike functions. J. Math. Anal. Appl. 145, 357-364 (1990)

17. Pashkouleva, DZ: The starlikeness and spiral-convexity of certain subclasses of analytic functions. In: Srivastava, HM, Owa, S (eds.) Current Topics in Analytic Function Theory, pp. 266-273. World Scientific, Singapore (1992)

18. Shams, S, Kulkarni, SR, Jahangiri, JM: Subordination properties of $p$-valent functions defined by integral operators. Int. J. Math. Math. Sci. 2006, Art. ID 94572 (2006)

19. Singh, R, Singh, S: Convolution properties of a class of starlike functions. Proc. Am. Math. Soc. 106, 145-152 (1989)

20. Srivastava, HM, Attiya, AA: An integral operator associated with the Hurwitz-Lerch Zeta function and differential subordination. Integral Transforms Spec. Funct. 18, 207-216 (2007)

21. Srivastava, HM, Choi, J: Series Associated with the Zeta and Related Functions. Kluwer Academic, Dordrecht (2001)

22. Stankiewicz, J, Stankiewicz, Z: Some applications of Hadamard convolution in the theory of functions. Ann. Univ. Mariae Curie-Sklodowska, Sect. A 40, 251-265 (1986)

23. Whittaker, ET, Watson, GN: A Course of Modern Analysis: An Introduction to the General Theory of Infinite Processes and of Analytic Functions; with an Account of the Principal Transcendental Functions, 4th edn. Cambridge University Press, Cambridge (1927)

24. Wilken, DR, Feng, J: A remark on convex and starlike functions. J. Lond. Math. Soc., Ser. 2 21, $287-290$ (1980)

doi:10.1186/1029-242X-2012-153

Cite this article as: Aouf et al.: Applications of differential subordinations for certain classes of $p$-valent functions associated with generalized Srivastava-Attiya operator. Journal of Inequalities and Applications 2012 2012:153. 